\title{
BIOSIGNALS-BASED AIR TREATMENT CONTROL SYSTEM FOR NEUROVEGETATIVE PATIENTS
}

\author{
Ștefan-Radu Bala, Ionuț Banu, Tania-Ioana Cîmpeanu, Bogdan-Gabriel Duță
}

Automatic Control, Computers and Electronics Department/Petroleum-Gas University of Ploiesti, Romania

e-mail: bogdangabriel_duta@yahoo.com

\section{DOI: 10.51865/JPGT.2021.02.06}

\begin{abstract}
The global epidemic of COVID-19 has highlighted a not obvious weakness of the medical system, namely establishing a favorable environment that can also contribute to the recovery process. This has led to many medical complications and the gravity of this situation could be mitigated by simply implementing an automatic system for monitoring and adjusting environmental parameters.

The paper highlights how the bio signals sampled by the sensors will be used for the calibration of the heating ventilation air conditioning control system and maintain the optimal parameters both in terms of temperature and humidity, which will prevent the development and transmission of microbes, while the ECG device and the EMG signal amplifier will help with the monitoring of the neurovegetative patients.
\end{abstract}

Keywords: control system, monitoring, bio signal, instrumentation amplifier, electrical activity.

\section{INTRODUCTION}

Because usually, in a hospital, the environmental parameters such as temperature, humidity and oxygen level are of a particular, but ignored importance, the proposed device aims to maintain their values at appropriate intervals for the patient's recovery [7,9].

Bio signals are biomedical signals that represent electrical signals obtained from organs in the human body. The cardiac cycle (cardiac revolution) includes the set of electromechanical events related to the passage of blood through the heart, during a cardiac systole and diastole. Systole is the periodic, rhythmic contraction of the heart muscle accompanied by the pushing of blood from the heart into the arteries. Diastole is the period of muscle relaxation and filling of the cardiac ventricles, following systole $[1,2,3]$.

The electromyogram (EMG) consists of recording the electrical activity of the muscles. When they are active, they produce electrical impulses directly proportional to the level of muscle activity. This procedure is an indispensable element in establishing a diagnosis in the pathology of the peripheral nervous system. EMG signals are used to detect abnormalities in muscle activity (myopathy - muscle atrophy, neuropathy disorder of the peripheral nervous system that manifests itself through muscle weakness, pain and numbness in the hands and feet). The electrical activity will be collected with surface electrodes, amplified, converted analog / digital and purchased by a computer $[2,4,5,8]$. 


\section{DEVICE PRESENTATION}

Conventional air handling systems typically have preset values or values that can be set by a human operator. The present paper aims to add to these devices by correlating the reference values with the vital signs of patients and to obtain a functional device for monitoring and amplifying EMG and EKG signals. This paper deals with the principle of construction of this type of medical equipment both through a systemic approach and by recommending its practical realization [7].

Brief description of the operation of the final device:

- regulation: depending on the vital signals, the environmental parameters will be regulated, such as: temperature, humidity, and oxygen level in the hospital;

- monitoring: vital signals will be captured: temperature, oxygen saturation in the blood, as well as electrocardiographic signals (monitoring of heartbeat and pulse) and electromyographic (monitoring of muscle activity).

The system for monitoring and adjusting the environmental parameters (figure 1) aims to bring and maintain the value of the output quantities to the set reference values. Establishment of reference values according to which quantities such as ambient temperature and relative humidity in the room to be adjusted should be adjusted according to the vital signs of the patient. The setting of the reference values will be performed automatically, without requiring direct human intervention [9].

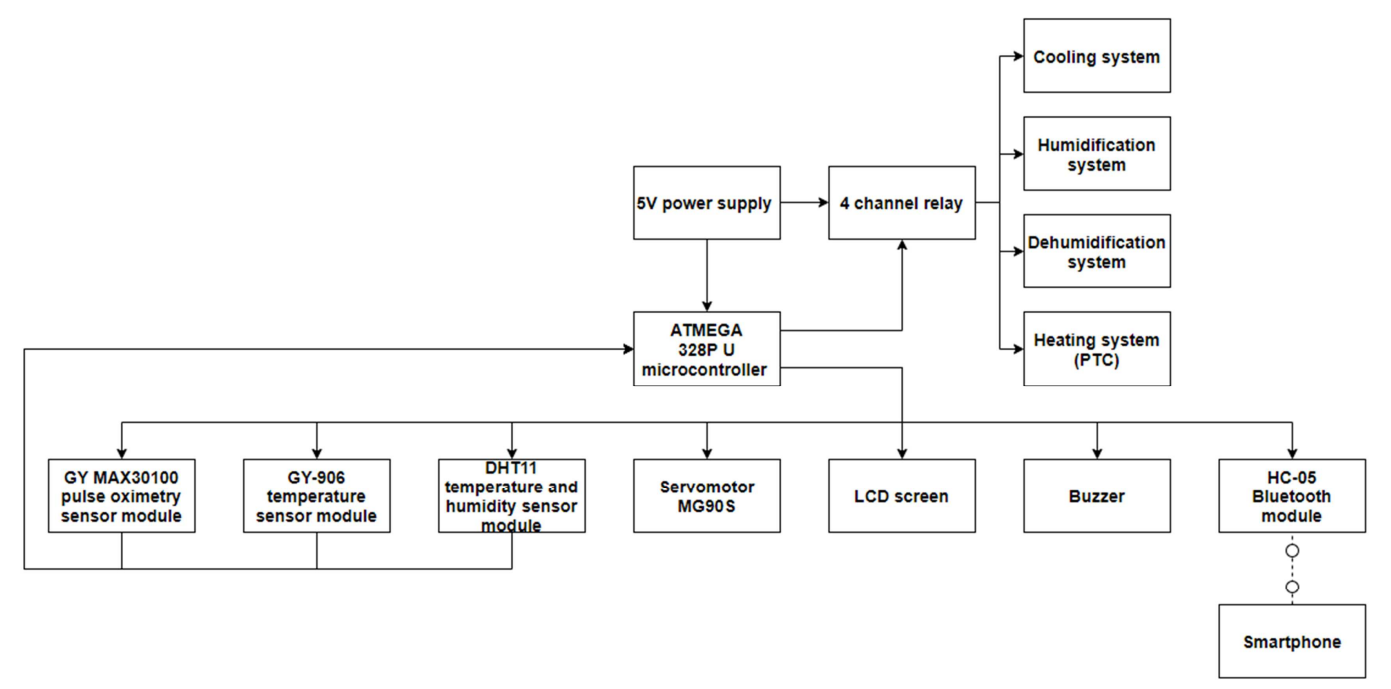

Figure 1. Block diagram of HVAC system connections

As can be seen in figure 2, for measuring the environmental parameters, it was opted for the DHT11 sensor module, which includes both a thermistor transducer for measuring temperature and a capacitive transducer for detecting the level of relative humidity in the air.

When measuring the patient's vital signs, the GY-MAX30100 optical pulse sensor module will be used to measure the level of oxygen saturation in the blood and the GY 906 wireless temperature sensor module to measure the patient's temperature constantly by a non-invasive method. 
The monitoring of the acquired data transmitted by the transducers will be performed by the ATMEGA 328P U microcontroller, depending on whose values the actuators will be activated, including: a fan that will circulate the air through a humid environment to increase the water vapor saturation in the air, a fan that will circulate the air through a dry environment to reduce the level of relative humidity in the air, a fan that will circulate the air through a cold environment to lower the temperature in the room, a mini heating element attached to a conductive surface that will release heat inside, all this being controlled by means of a four-channel relay and an MG90S servomotor that will actuate the reducing clock of the oxygen tube, simulated in the work by means of a compressed air spray.

The system has been designed so that both the environmental parameters and the vital signs of the patient are displayed on the screen of the phone connected via Bluetooth technology, along with the reference values obtained. At the same time, if the medical staff will have to be alerted, the buzzer will be activated.

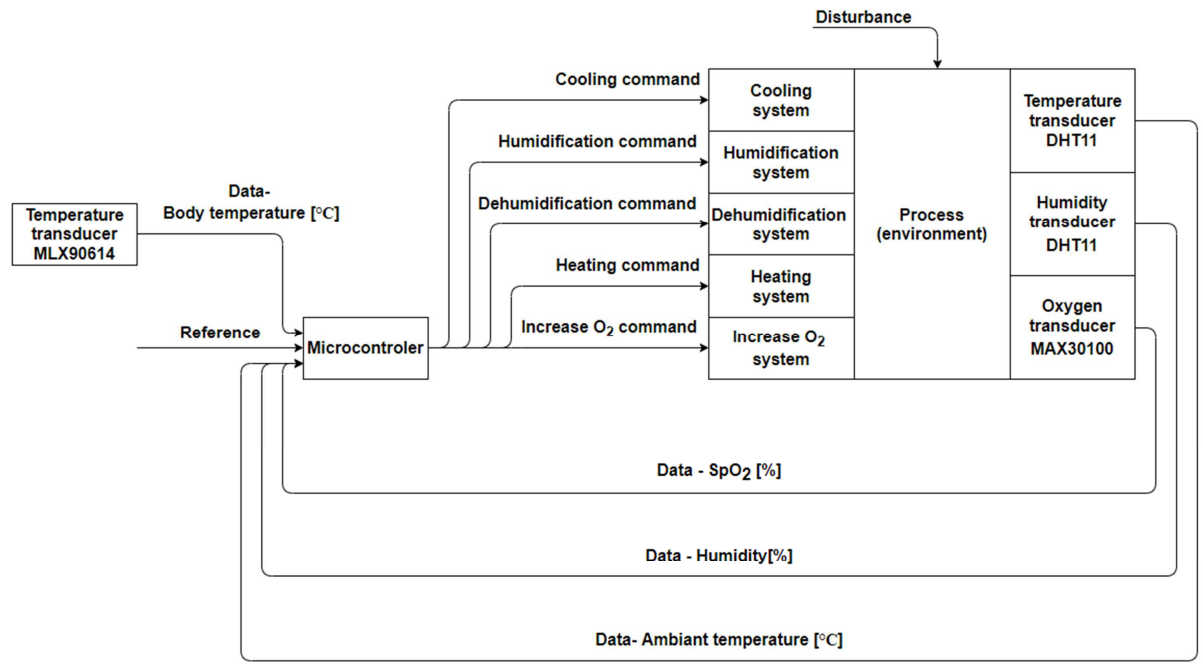

Figure 2. Functional block diagram of the Heating Ventilation Air Conditioning system

The EKG signal consists of the overlapping of the potential differences generated by the heart. EKG devices use electrodes to convert ionic signals in the body into electrical signals for display and analysis. In the case of the system for measuring and converting bioelectric signals from the heart muscle, the EKG-EMG-PA electrodes were used as sensors. They are located on the left arm, right arm and right leg. The electrodes are connected via the adapter to the OLIMEX EKG-EMG module, as seen in figure 3 [7].

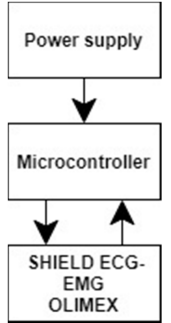

Figure 3. Scheme of system connections of the ECG system 
Because the variations of the EKG signal are of the order of microvolts and millivolts, its measurements must be amplified. This role belongs to the OLIMEX EKG-EMG module, being an instrumentation amplifier followed by an operational amplifier and a low pass filter. The module uses amplifiers to accurately intensify the differential voltages measured with the electrodes attached to the subject's body (figure 4). The differential analog signal is converted by the EKG module, set to the input channels CH1_IN+ / CH1_IN-, into a single amplified and filtered analog signal stream. The signal thus obtained is sent to the ATmega328 microcontroller, which analyzes the values received in order to detect irregularities in the heart rhythm [7].

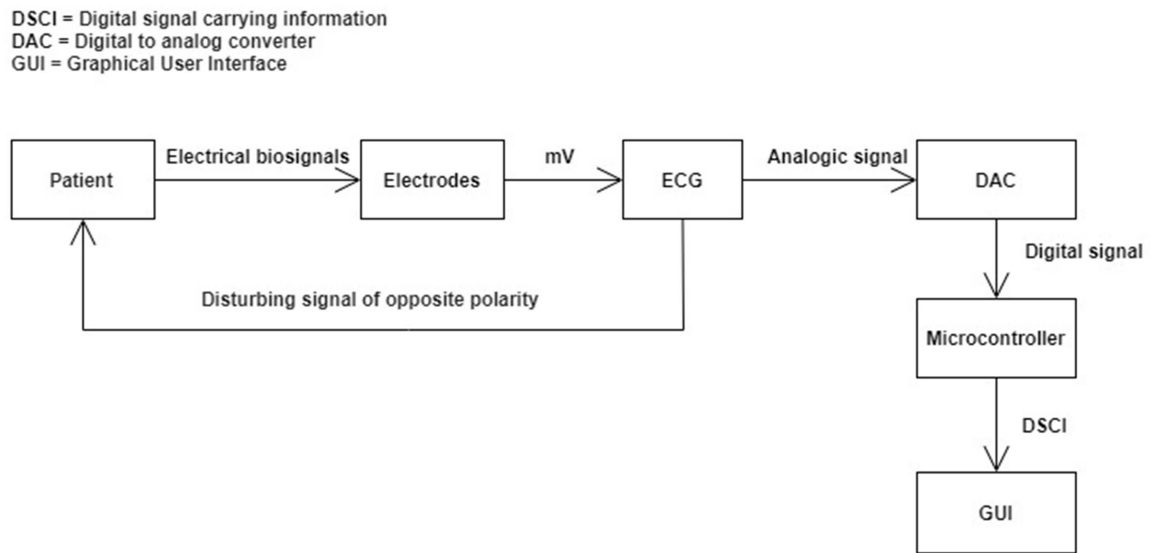

Figure 4. Functional block diagram of the ECG device

The system consists of two parts:

- controller (implemented by a software program): the digital potentiometer works at the software control;

- signal conditioner (instrumentation amplifier): that amplifies the signals received from the muscles.

Between the transducer and the regulator there are signal conditioners (it conditions the signal, processes it to bring it to the level at which the automatic system can understand, accept and optimize it - the signal / noise ratio). The main element of the system (core) is the instrumentation amplifier (AI). The output of the AI (amplified EMG signals) represents the input of the ADC (analog-to-digital converter). The signals are converted so that they can be taken over by the controller (here, the controller is the software application). The command to change the cursor of the digital potentiometer (adjustment direction - up / down) is given from the controller (the program written in Arduino).

In order to achieve the system, the following components were used: LT1167 instrumentation amplifier; AD8402 digital potentiometer; two LT1112 operational amplifiers; Adafruit EMG surface electrodes; Arduino Uno Rev3 development board; resistors with metal film of different values: $30 \mathrm{k}, 12 \mathrm{k}, 10 \mathrm{k}, 1 \mathrm{k}, 1 \mathrm{M}$; polypropylene capacitors of different values: $0.01 \mu, 0.47 \mu, 15 \mathrm{n}$; breadboard; fire jumper; $3.5 \mathrm{~mm}$ stereo audio cable with angular connector.

The block diagram of the EMG signal amplifier is presented in figure 5. 


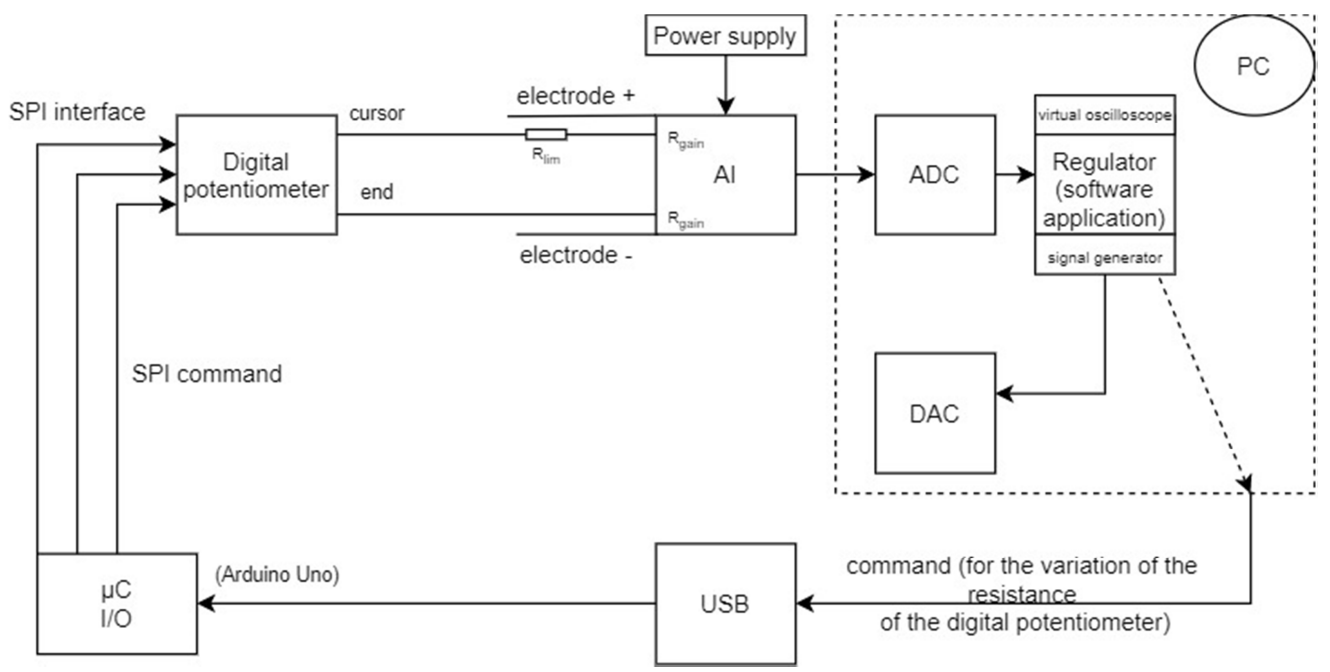

Figure 5. Block diagram of the EMG signal amplifier

\section{EXPERIMENTAL RESULTS}

The volume of oxygen retained by the body in one minute at rest is $200-250 \mathrm{ml} / \mathrm{min}$, and it increases up to 20 times more during physical exertion.

At the same time, the volume of the salon will be considered $30 \mathrm{~m} 3(\mathrm{~L}: 4 \mathrm{~m}, 1: 3 \mathrm{~m}$, h: $2.5 \mathrm{~m}$ ), the equivalent of 30,000 liters, to determine the air flow to be released and a corresponding oxygen tube.

Determination of the volume of oxygen in the chamber, considering the percentage of oxygen at the level of $20 \%$, that is $20 \% * 300001=60001$, means that, on average, a person consumes about $250 \mathrm{ml} / \mathrm{min}$, the equivalent of $161 /$ hour. Consequently, to increase the level of oxygen in the air in the living room by $1 \%$ implies 3081 of pure oxygen $(3001+81)$ which will have to be released for 30 minutes, i.e. a minimum of $10.81 /$ minute.

The experimental results for air heating / cooling and humidification / dehumidification processes, presented in the following tables, point out the slow trend of adjustment, which in the medical field is an asset due to the minimal stress of body accommodation, the system thus fulfilling its role of monitoring and adjusting environmental parameters in a patient recovery room.

Table 1. Experimental results of the heating system

\begin{tabular}{|c|c|c|c|}
\hline No & Time [minutes] & Reference $\left[{ }^{\circ} \mathrm{C}\right]$ & Measurement $\left[{ }^{\circ} \mathrm{C}\right]$ \\
\hline 1 & 0 & 23 & 20 \\
\hline 2 & 10 & 23 & 20 \\
\hline 3 & 20 & 23 & 21 \\
\hline 4 & 30 & 23 & 22 \\
\hline 5 & 40 & 23 & 22 \\
\hline 6 & 50 & 23 & 23 \\
\hline 7 & 60 & 23 & 23 \\
\hline
\end{tabular}


Table 2. Experimental results of the cooling system

\begin{tabular}{|c|c|c|c|}
\hline No & Time [minutes] & Reference $\left[{ }^{\circ} \mathrm{C}\right]$ & Measurement $\left[{ }^{\circ} \mathrm{C}\right]$ \\
\hline 1 & 0 & 19 & 22 \\
\hline 2 & 10 & 19 & 21 \\
\hline 3 & 20 & 19 & 21 \\
\hline 4 & 30 & 19 & 20 \\
\hline 5 & 40 & 19 & 19 \\
\hline 6 & 50 & 19 & 19 \\
\hline 7 & 60 & 19 & 19 \\
\hline
\end{tabular}

Table 3. Experimental results of the humidification system

\begin{tabular}{|c|c|c|c|}
\hline No & Time [minutes] & Reference $\left[{ }^{\circ} \mathrm{C}\right]$ & Measurement $\left[{ }^{\circ} \mathrm{C}\right]$ \\
\hline 1 & 0 & 40 & 37 \\
\hline 2 & 10 & 40 & 37 \\
\hline 3 & 20 & 40 & 38 \\
\hline 4 & 30 & 40 & 39 \\
\hline 5 & 40 & 40 & 39 \\
\hline 6 & 50 & 40 & 40 \\
\hline 7 & 60 & 40 & 40 \\
\hline
\end{tabular}

Table 4. Experimental results of the dehumidification system

\begin{tabular}{|c|c|c|c|}
\hline No & Time [minutes] & Reference $\left[{ }^{\circ} \mathrm{C}\right]$ & Measurement $\left[{ }^{\circ} \mathrm{C}\right]$ \\
\hline 1 & 0 & 35 & 39 \\
\hline 2 & 10 & 35 & 38 \\
\hline 3 & 20 & 35 & 38 \\
\hline 4 & 30 & 35 & 37 \\
\hline 5 & 40 & 35 & 36 \\
\hline 6 & 50 & 35 & 35 \\
\hline 7 & 60 & 35 & 35 \\
\hline
\end{tabular}

The electrocardiogram (ECG) represents the graphical recording of the potential differences generated during the cardiac electrical activity, detecting irregularities of the heart rhythm. The ECG helps monitor, evaluate and diagnose the patient.

The waves on an EKG include the $\mathrm{P}$ wave, the $\mathrm{Q}$ wave, the $\mathrm{R}$ wave, the $\mathrm{S}$ wave, the $\mathrm{T}$ wave, and the $\mathrm{U}$ wave.

The $\mathrm{P}$ wave represents atrial depolarization (in healthy individuals, the $\mathrm{P}$ wave precedes each QRS complex. The PR interval extends from the beginning of the $\mathrm{P}$ wave to the beginning of the QRS complex, representing the time required for electrical activity to move between the atria and ventricles. The QRS complex represents ventricular depolarization and consists of 3 close waves: Q, R and S. The ST segment begins at the end of the QRS complex and stops at the beginning of the T wave, representing the time between depolarization and ventricular repolarization (called ventricular contraction) [6].

The $\mathrm{T}$ wave (ventricular repolarization) is a small wave that occurs after the QRS complex [6]. 
The interval RR starts from the vertex of one wave $\mathrm{R}$ and ends at the vertex of the next wave $\mathrm{R}$ and represents the time between two QRS complexes [6].

The QT interval extends from the beginning of the QRS complex to the end of the T wave and represents the time required for depolarization and ventricular repolarization.

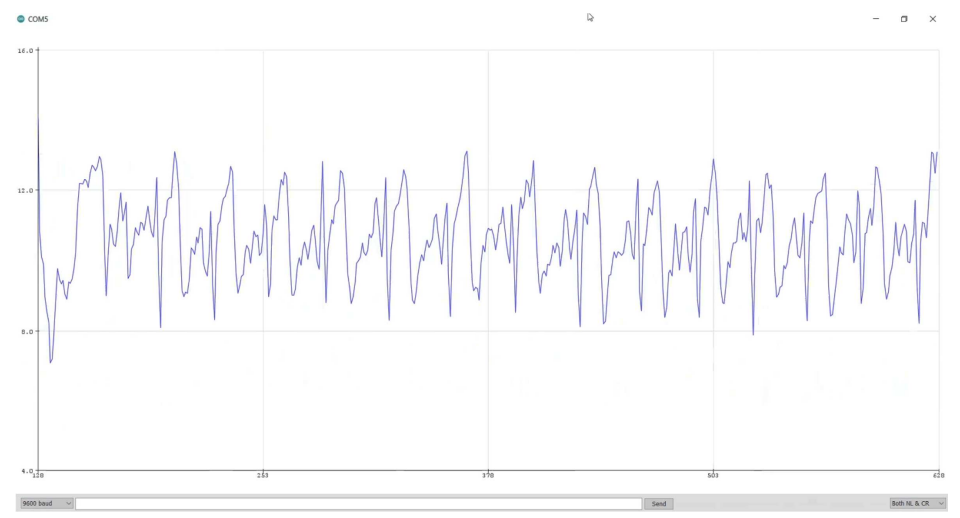

Figure 6. The waveform of the electrocardiographic signal associated to first set values

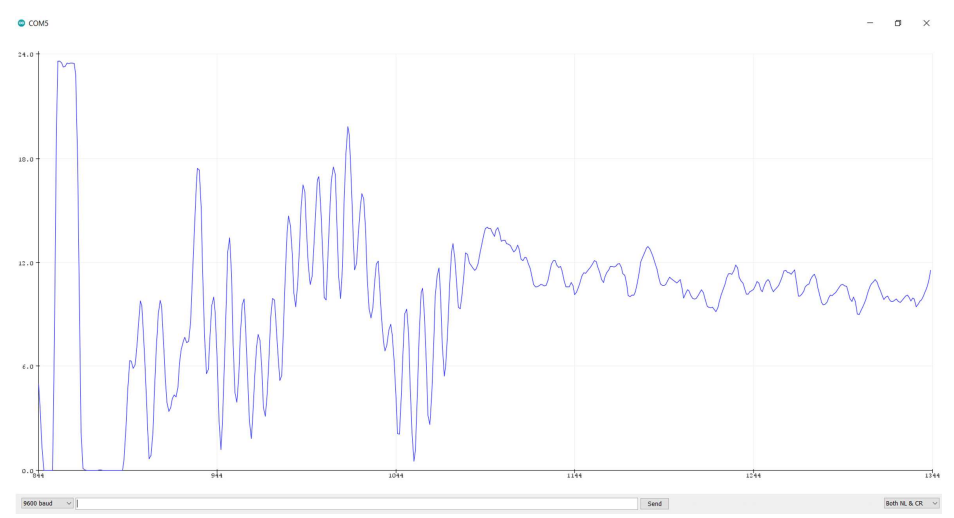

Figure 7. Waveform of the electrocardiographic signal specific to cardiac dysfunctions

A set of 10 experimental data was entered (Figure 8) and then the gain was calculated using the formula taken from the catalog sheet for the LT1167 instrumentation amplifier (Figure 9) [10].

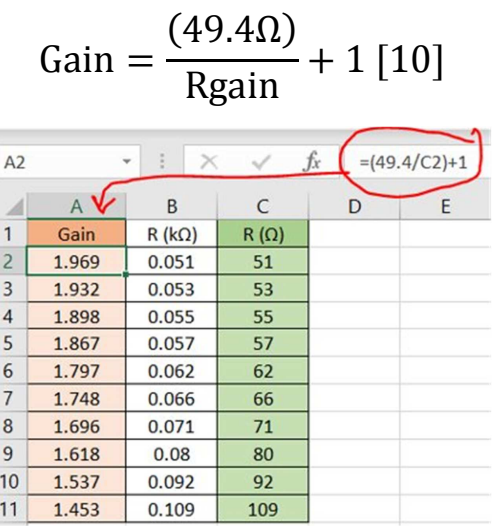

Figure 8. Experimental data set 


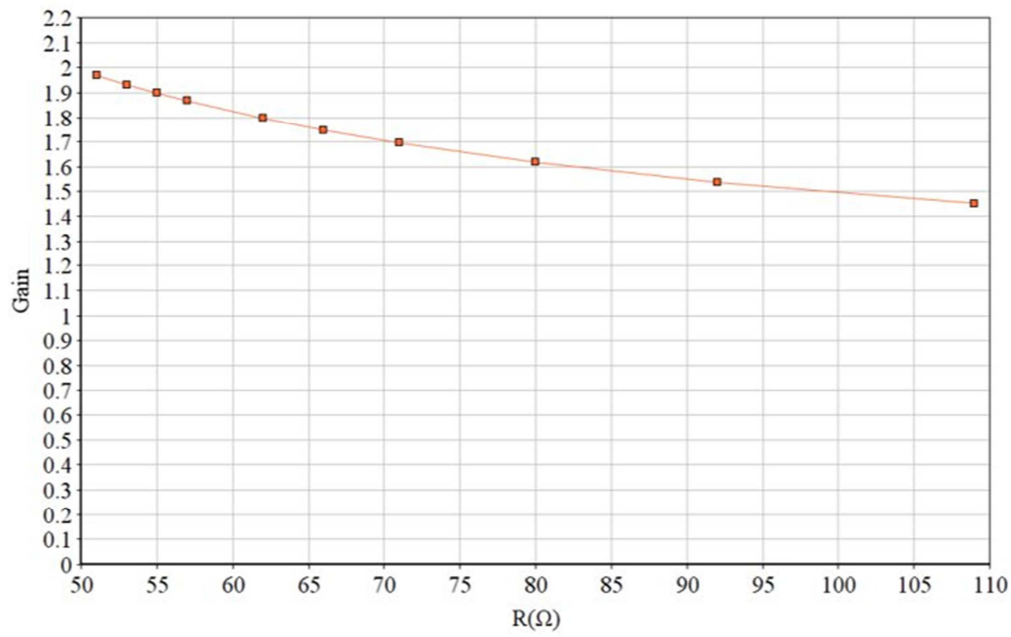

Figure 9. Dependency graph gain $=f(R$ gain $)$

One can observe that as the value of the variable resistor increases, the value of the gain decreases (the gain is variable, depending on the values of the variable resistor).

Below are presented some graphs showing the amplified muscle activity, that can be further interpreted in order to find a diagnosis. The muscular activity of two patients was monitored, as seen in Figure 10 and Figure 11; the EMG signals are directly proportional to the muscular activity of the two patients.

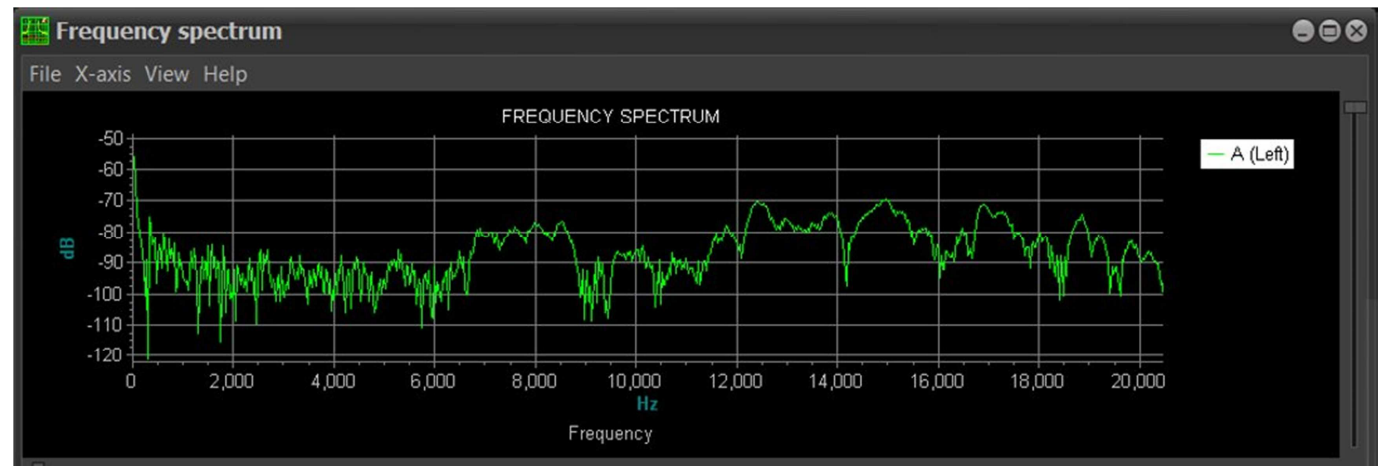

Figure 10. EMG signal in the frequency spectrum (easy muscle relaxation)

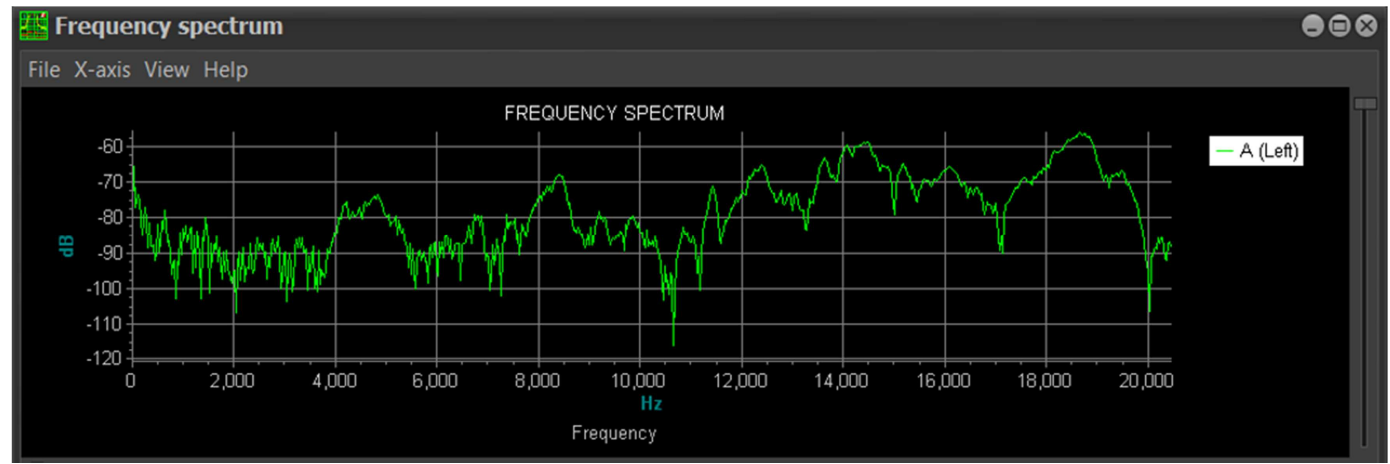

Figure 11. EMG signal in the frequency spectrum (high intensity muscle activity) 


\section{CONCLUSION}

The proposed device aims to obtain a monitor of the condition of a patient with diseases of the neurovegetative system and the regulation of environmental parameters in the living room, all of which are powered by renewable energy.

By implementing this automatic device for monitoring and adjusting environmental parameters, it is possible to prevent the occurrence of diseases or infections such as nosocomial. It will be able to maintain the optimal parameters both in terms of temperature and humidity, which will prevent the development and transmission of microbes.

The role of the instrumentation amplifier is to facilitate any form of measurement due to its accuracy, the fact that it has an infinite number of states (because it is an analog system) and immunity to noise.

Instrumentation amplifier is used in any form of instrumentation or interface with transducers, for any kind of automated system (monitoring, measurement, processing).

The amplitude of the muscle activity signal does not always have the same value, depending on the type of activity that the patient does during the EMG procedure. Each person has a different amplitude of the signal of muscle activity, this can be influenced by the type of electrodes used (invasive / non-invasive), their placement in / on the muscles and the texture of the muscle surface.

As future research directions, one must consider the fact that EMG signals can be used for a variety of applications such as clinical or biomedical applications.

A very important field is that with reference to the development of EHW chips, namely evolvable hardware, that aims to use evolutionary algorithms to produce specialized electronics without manual engineering, bringing together reconfigurable hardware, artificial intelligence, evolutionary calculus and adaptive systems.

To this effect, EMG signals can be used in the development of EHW chips for prosthetic hand control and detection of isometric muscle activity (a type of muscle activity that cannot be translated by movement), thus making it possible to define a class of subtle gestures for controlling an interface that can be observed without disturbing the environment.

\section{REFERENCES}

[1] Bala Ș., Inginerie biomedicală, Note de curs, UPG Ploiești, 2019.

[2] Hariton N., Electronică medicală, Editura Cantes, Iasi, 2000.

[3] Hudgins B., Parker P., Scott SH.: A new strategy for multifunction myoelectric signals, IEEE Trans Biomed Eng, 1993.

[4] Luca C.J., The use of surface electromyography in biomechanics, J Appl Biomech, 1997.

[5] Reaz M.B., Hussain M.S., Mohd-Yasin F., Techniques of EMG signal analysis: detection, processing, classification and applications, Biol Proced Online, 2006.

[6] Saint Amant Y., Rancourt D., Clancy E.A., Influence of Smoothing Window Length on Electromyogram Amplitude Estimates, IEEE Transactions On Biomedical Engineering, 1998. 
[7] Sehulster L., Chinn R.Y., Guidelines for environmental infection control in health-care facilities, Recommendations of CDC and the Healthcare Infection Control Practices Advisory Committee (HICPAC), MMWR Recomm Rep, 2003.

[8] https://www.smithsonianmag.com/history/when-fresh-air-went-out-fashionhospitals-180963710/

[9] https://www.uofmhealth.org/health-library/hw198785

[10] https://www.analog.com/media/en/technical-documentation/datasheets/1167fc.pdf 